\title{
RESÚMENES DE TRABAJOS ORALES
}

\section{Miércoles 28}

\section{2:10-12:40}

01

12:10-12:20
Inferencia sobre la afectación del programa de donación del Hospital General Celaya por la suspensión de los programas de donación debido al virus SARS-COV-2

Martínez Bernal Salvador, Colio Montoya Martha María,

Gutiérrez Servín María Silvia.

Hospital General Celaya, Centro Estatal de Trasplantes Guanajuato.

Introducción: El Hospital General Celaya es un hospital donante desde septiembre de 2015, con una tasa de donación de 21.74 donantes por millón de población (PMP) y tasa de conversión de donantes de $55.49 \%$. El 19 de marzo de 2020, debido al virus SARS-COV-2, se suspenden los programas de donación y trasplante, con lo cual la actividad del hospital cae a cero. Para conocer el impacto en donación, se realiza un análisis de las defunciones ocurridas en el periodo del 19 de marzo al 31 de julio de 2020. Material y métodos: Se realiza un análisis retrospectivo, utilizando una base de datos creada en Access, registrando las defunciones ocurridas en el periodo estudiado con los siguientes datos; ID del paciente, edad, diagnóstico, sala de defunción, paciente neurocrítico (sí/no).

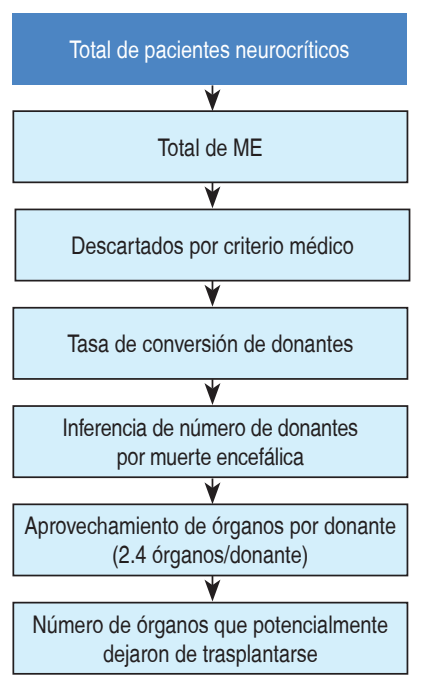

Resultados: Se registró un total de 315 defunciones en el periodo. De éstas, se contabilizaron 43 defunciones de neurocríticos, se realizó el diagnóstico de muerte encefálica a seis, descartando un paciente con sospecha de COVID-19, para un total de cinco potenciales donantes, el cual corresponde a $12.2 \%$ de los neurocríticos. Para la tasa de conversión de donantes (55.49\%), se esperarían tres donantes efectivos. Respecto a posibles donantes de tejidos, se tuvieron 309 defunciones en parada cardiaca, descartándose 161 por diagnóstico confirmado o sospecha de COVID-19, quedando un total de 148 posibles donantes de tejidos. Aplicando el indicador de donantes de tejidos esperados (5\%), se habrían esperado siete donantes de tejidos efectivos. El aprovechamiento en nuestro hospital es de 2.4 órganos/donante y 2.7 tejidos/donante, esto significa que se perdieron aproximadamente 7.2 órganos en el caso de donantes en muerte encefálica y 18.9 tejidos para donantes en parada cardiaca, para un total de 26.1 órganos y tejidos en el periodo estudiado.

\begin{tabular}{|c|c|c|c|}
\hline Indicadores ME & & Indicadores PC & \\
\hline Total de defunciones & 315 & Total de defunciones & 309 \\
\hline$\%$ de ME en áreas críticas & 12.2 & $\%$ esperado de donantes & 5 \\
\hline Total de ME & 6 & $\begin{array}{l}\text { Descartados por } \\
\text { COVID o sospecha }\end{array}$ & 161 \\
\hline $\begin{array}{l}\text { Descartados por } \\
\text { contraindicación médica }\end{array}$ & 1 & $\begin{array}{l}\text { Total de defunciones } \\
\text { consideradas }\end{array}$ & 148 \\
\hline $\begin{array}{l}\text { \% conversión de donantes } \\
\text { (ME/concretados) }\end{array}$ & 55.4 & Donantes esperados & 7 \\
\hline Aprovechamiento órganos/donante & 2.46 & $\begin{array}{l}\text { Aprovechamiento } \\
\text { tejidos/donante }\end{array}$ & 2.7 \\
\hline Órganos no procurados & 7.2 & Tejidos no procurados & 18.9 \\
\hline
\end{tabular}

Discusión: Se evalúo el impacto de la ausencia de actividad aplicando los indicadores de calidad, lo cual arrojó la pérdida de 7.2 órganos y 18.9 tejidos como inferencia. Conclusiones: Este trabajo pretende evaluar el impacto al programa de donación en nuestro hospital, midiendo de manera objetiva y con indicadores bien establecidos el número aproximado de donantes perdidos, así como el número aproximado de órganos y tejidos que dejaron de beneficiar al mismo número de receptores en lista de espera. Esperamos que este documento sirva como guía para que otras coordinaciones de donación evalúen el impacto de la pandemia en sus hospitales.
O2

12:20-12:30
Utilidad del I-IFTA en biopsias protocolarias del tercer mes y al año como factor pronóstico de supervivencia del injerto renal en comparación con marcadores indirectos de la función renal en pacientes trasplantados mexicanos en el Centro Médico Nacional 20 de Noviembre 
Matías Carmona Mayra, * Cano Cervantes José Horacio,, Hernández Estrada Sergio, ${ }^{\ddagger}$ Díaz Avendaño Odette del Carmen, ${ }^{\ddagger}$ Torres Pastrana Juvenal, ${ }^{*}$ Hernández Hernández Regina Canade ${ }^{\S}$

* Departamento de Nefrología. ${ }^{\ddagger}$ Unidad de Trasplante. ${ }^{\S}$ Servicio de

Patología. ${ }^{3}$ Centro Médico Nacional "20 de Noviembre», CDMX, México.

Introducción: El trasplante renal se ha convertido en la primera opción de tratamiento para enfermedad renal crónica terminal; sin embargo, existen múltiples factores que condicionan pérdida de la función del injerto; para lo cual se han implementado estrategias diagnósticas de seguimiento en el postrasplante. Recientemente se ha demostrado que patrones histológicos como i-IFTA son útiles para evaluar la supervivencia del injerto en biopsias protocolarias. Objetivo: Comparar la utilidad del i-IFTA contra los marcadores indirectos de la función renal, medidos al tercer mes y al año como factor pronóstico de supervivencia del injerto en pacientes mexicanos sometidos a trasplante renal. Material y métodos: Se incluyeron 74 pacientes trasplantados de injerto renal a los cuales se les realizaron biopsias protocolarias a los tres y 12 meses postrasplantes; 26 (25.4\%) mujeres, edad promedio fue de $35.6 \pm 10$ años. Se dividió a la población en dos subgrupos; con y sin rechazo; se evaluó la utilidad de i-IFTA en biopsias protocolarias a los tres y 12 meses postrasplante como factor pronóstico de supervivencia del injerto renal, comparado con marcadores indirectos de la función renal: creatinina y albuminuria en orina de 24 horas. Resultados: No se observaron diferencias entre grupos según las características demográficas, al evaluar el rendimiento diagnóstico de albuminuria y creatinina sérica a los tres meses respecto al evento inmunológico, no se encontró significancia estadística $(p=0.08)$; sin embargo, se demostró la progresión de dichos marcadores en las biopsias anuales, presentando mayor grado de albuminuria y niveles séricos de creatinina los subgrupos con grado histológico desfavorable, catalogado en nuestro estudio como i-IFTA $>1$. Se observó que la persistencia de i-IFTA 1 en las biopsias anuales confiere protección para evento inmunológico, comparado con los otros subgrupos histológicos. En las variables de seguimiento se encontró diferencia significativa entre las categorías de ADES entre grupos; los pacientes sin rechazo mostraron menor proporción de ADES comparados con el grupo que presentó evento inmunológico. Conclusiones: El rendimiento de i-IFTA en biopsias protocolarias a los tres meses es pobre comparado con las biopsias anuales. La asociación de ADES con MFI > 4000 asociado a i-IFTA $>1$ confiere riesgo para evento inmunológico. Se requiere completar una muestra más grande y un seguimiento más prolongado para evaluar el i-IFTA como factor pronóstico de supervivencia del injerto renal y así evaluar la rentabilidad de los marcadores indirectos de la función renal; albuminuria y creatinina sérica.

\section{Eventos inmunológicos}

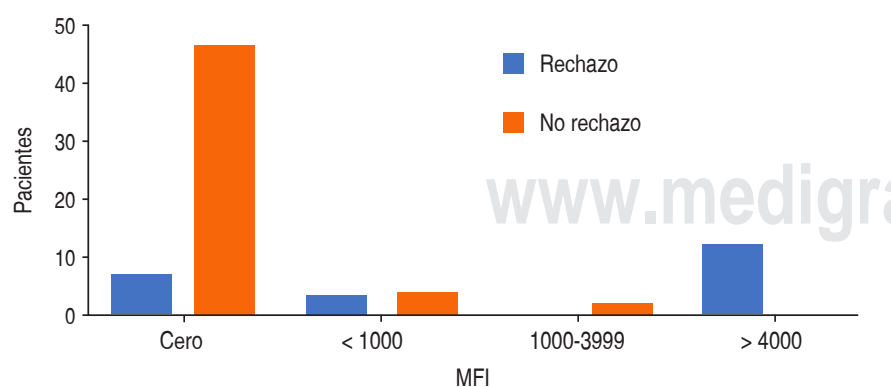

Figura 1: Eventos inmunológicos de acuerdo a subgrupos de título de anticuerpos por intensidad de fluorescencia media.

MFI = Título de anticuerpos por intensidad de fluorescencia media.

\section{3}

12:30-12:40

Estrategias para mejorar el entrenamiento de los cirujanos de trasplante en formación, durante la pandemia por SARS-CoV-2

Vázquez Salinas Carlos, Florez Zorrilla Carlos, Buganza Torio Elizabeth, Charco Cruz Miguel, Romero Beyer Nadia A, De Jesús Flores Alberto, Olivares del Moral Josué I, Núñez Venzor Alejandra, Sánchez Cedillo Aczel I Centro Médico Nacional 20 de Noviembre, ISSSTE.

Introducción: La cirugía es una disciplina médica orientada a la acción. Formar excelentes cirujanos requiere procesos de enseñanza y aprendizaje explícitos y predecibles en sus resultados. La educación en cirugía no debe estar basada solamente en una técnica quirúrgica, va mucho más allá. El fellow de trasplantes considera que sus habilidades y conocimientos sólo deben basarse en el número de procedimientos quirúrgicos que realice y vea, aunque es importante, no es lo fundamental. El cirujano de trasplantes debe adquirir una serie de conocimientos en medicina interna, nefrología, hepatología, terapia intensiva, que lo lleven a desempeñarse apropiadamente en situaciones clínicas y quirúrgicas variadas que lo lleven a un excelente juicio y toma de decisiones. En la actualidad, el mundo vive una pandemia de coronavirus SARS-CoV-2, situación que ha puesto en serios conflictos a todos los sectores, principalmente a los trabajadores de la salud, que día a día enfrentan una batalla contra la enfermedad y la muerte. Los trasplantes han sido duramente castigados por esta pandemia, repercutiendo en la salud de los pacientes en lista de espera de algún órgano o tejido, también se ha visto afectado el entrenamiento de los cirujanos de trasplante en formación. Con el presente trabajo queremos establecer pautas o estrategias para el mejoramiento de la formación de los cirujanos de trasplante en esta nueva normalidad. Material y métodos: Se elaboró un cuestionario de 10 preguntas en el que aleatoriamente se entrevistó a 30 individuos que incluyeron fellows o residentes de cirugía de trasplantes de primer año y de segundo año, profesores titulares de curso, adscritos al servicio de trasplantes y adscritos de hepatología, en el que se establecieron las medidas para mejorar la enseñanza de forma segura de los residentes durante la pandemia de COVID-19. De lo establecido en el cuestionario se realizó una revisión en la literatura en la que se definió la seguridad de las medidas sugeridas por el personal de un Servicio de Trasplantes. Resultados: De los 30 entrevistados, se llegaron a las siguientes conclusiones, las principales respuestas fueron que hasta un $70 \%$ piensa que se puede regresar a la actividad de trasplantes, una vez que mejoren las condiciones generales de los hospitales trasplantadores, $80 \%$ considera que es necesario regresar a laboratorios de cirugía experimental para reactivar las habilidades quirúrgicas de los residentes, $90 \%$ de los entrevistados considera que es necesario contar con respiradores $\mathrm{N} 95$, o en su defecto respiradores 3M, así como EPP y que en las salas de quirófano se cuente con máquinas de presión negativa y adecuada ventilación para sentirse seguros durante un trasplante. El $80 \%$ de los entrevistados considera que se debe mantener el entrenamiento de los residentes, para que puedan desarrollar sus competencias. El $100 \%$ de los entrevistados considera pertinente realizar PCR y TAC de tórax a los pacientes y donadores de órganos, así como anticuerpos al personal de salud de forma frecuente para evitar contagios. Un $70 \%$ de los casos considera útil el espaciamiento de los días entre todo el personal para evitar aglomeraciones y evitar más contagios entre el personal de salud. La mayoría consideró pertinente, una vez terminada la contingencia, realizar trasplantes diariamente para retomar la actividad previa a la pandemia. Discusión y conclusiones: Esta pandemia le ha dado un giro a la enseñanza quirúrgica, ya que durante este periodo todos los esfuerzos de los servicios quirúrgicos se concentrarán en proporcionar la mejor atención posible a la población afectada por el COVID-19, al mismo tiempo que se protege y preserva la fuerza laboral quirúrgica. La educación quirúrgica no debe ser abandonada. La educación quirúrgica virtual debe considerarse una opción para mitigar 
el efecto que ha tenido esta pandemia en el entrenamiento de los cirujanos de trasplante. Existe una preocupación racional sobre el riesgo que implica que los residentes de cirugía de trasplantes regresen a los quirófanos; sin embargo, día con día se conocen más los efectos del virus en los humanos, así como la forma de evitar su propagación. Entre las estrategias que se cuentan están el uso de elementos de protección personal (EPP) obligatorios para todos los médicos residentes en rotaciones clínico-quirúrgicas con riesgo de exposición a pacientes COVID-19 positivos, mantener un distanciamiento social, uso obligatorio de cubrebocas, el apoyo en los residentes de mayor jerarquía para la educación de los residentes de nuevo ingreso para atenuar ausencias del personal de base, así como el uso de plataformas para la difusión de la información evitando el contacto entre personal de salud.

\section{4}

Impacto de COVID-19 en la función del

$12: 40-12: 50$ injerto renal: estudio multicéntrico

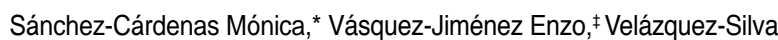
Ricardo Iván, " Vilatobá-Chapa Mario,§ Gómez-Navarro Benjamín," Sánchez-Macías Lucio Octavio," Rodríguez-Chagolla José Manuel, ** García-Juárez Ignacio,

Flores-Gama César, ${ }^{*}$ Parra-Ávila Idalia, "ף Morales-Buenrostro Luis Eduardo* * Nefrología del Trasplante. Departamento de Nefrología y Metabolismo Mineral. INCMNSZ. ‡ Departamento de Nefrología, INCICh. ${ }^{\circledR}$ Departamento de Trasplante, INCMNSZ. " Departamento de Nefrología. CMNO, IMSS. Guadalajara. " Hospital San Javier, Guadalajara, México. ** Departamento de Nefrología y Medicina Interna. Centro Médico ISSEMyM, Toluca. \# Departamento de Gastroenterología. INCMNSZ. ${ }^{\$}$ Departamento de Trasplante. UMAE, Centro Médico Nacional del Bajío. " Departamento de Trasplante. UMAE, IMSS. Mérida, Yucatán, México.

Introducción: Los receptores de trasplante renal, dado su alto nivel de comorbilidad y exposición a inmunosupresión crónica, se encuentran expuestos a desenlaces adversos al exponerse a cualquier evento infeccioso. Particularmente en el caso de la infección por COVID-19 con gran involucro sistémico, el injerto renal enfrentará múltiples retos que pondrán a prueba su reserva funcional, con riesgo incrementado de lesión renal aguda. Material y métodos: Evaluamos de manera retrospectiva aquellos receptores de trasplante renal mayores de 18 años, con diagnóstico confirmado de infección por COVID-19 tratados en seis centros de tercer nivel en México. Resultados: Los datos de 45 receptores de trasplante renal fueron registrados. La mediana de edad global fue de 45 años (25-70). El $26.7 \%$ era portador de diabetes mellitus y $60 \%$ tenía hipertensión arterial. La mediana de tiempo postrasplante fue de 71 meses (IQR 40180). Fue requerido proceder a hospitalización en sala general en 26 casos $(57.8 \%)$, con ingreso a la Unidad de Terapia Intensiva en ocho casos $(17.8 \%)$. Once (24.4\%) pacientes recibieron manejo ambulatorio. Se documentó lesión renal aguda en 30 casos (66.6\%), de los cuales 18/30 (60\%) fueron AKI 2 o 3 . Seis pacientes tuvieron requerimientos dialíticos, de los cuales $4 / 6$ tuvieron recuperación completa de la función renal con egreso posterior. La mortalidad global fue de $13.3 \%(n=6)$. De los pacientes que murieron, $1 / 6$ falleció con injerto en su función renal basal, $1 / 6$ con lesión AKIN 1 y cuatro pacientes con AKIN 3, 2/4 requirieron terapia de reemplazo renal. El desenlace renal posterior de los pacientes supervivientes se muestra en la Tabla 1. Ningún paciente egresó dependiente de terapia dialítica. La mediana de días de hospitalización fue de ocho (IQR 6-12) días. Cinco pacientes continúan hospitalizados al momento. Discusión y conclusión: En nuestro grupo de pacientes el desarrollo de lesión renal aguda fue frecuente, estuvo presente en 2/3 de los individuos. Sin embargo, se registró recuperación del filtrado glomerular en la gran mayoría de los casos. Los factores predisponentes a deterioro renal pudieran ser similares a lo reportado en la población general, con necesidad de estudios de mayor dimensión para establecer tales determinantes. Consideramos que el manejo multidisciplinario resulta crucial para alcanzar desenlaces favorables.

Tabla 1 (04): Características basales y evolución clínica de los receptores de trasplante renal según la presencia de lesión renal aguda $(\mathrm{N}=45)$.

\begin{tabular}{|c|c|c|c|c|}
\hline & $\begin{array}{c}\text { Sin lesión } \\
n=15 \\
(33.3 \%)\end{array}$ & $\begin{array}{c}\text { AKI 1 } \\
n=12 \\
(26.7 \%)\end{array}$ & $\begin{array}{c}\text { AKI } 2 \\
n=6 \\
(13.3 \%)\end{array}$ & $\begin{array}{c}\text { AKI } 3 \\
n=12 \\
(26.7 \%)\end{array}$ \\
\hline \multicolumn{5}{|c|}{ Características clínicas y demográficas, $n(\%)$} \\
\hline Edad, mediana [IQR] & 50 [43-62] & $\begin{array}{c}39.5 \\
{[(29.2-52.7]}\end{array}$ & $\begin{array}{c}42 \\
{[30.5-48]}\end{array}$ & $\begin{array}{c}42.5 \\
{[32.7-53]}\end{array}$ \\
\hline Género masculino & $7(46.7)$ & $7(58.3)$ & $3(50.0)$ & $9(75.0)$ \\
\hline \multicolumn{5}{|l|}{ Comorbilidad } \\
\hline Diabetes & $5(33.3)$ & $4(33.3)$ & $1(16.7)$ & $2(16.7)$ \\
\hline Hipertensión & $9(60.0)$ & $5(41.7)$ & $3(50.0)$ & $10(83.3)$ \\
\hline Sobrepeso & $5(33.3)$ & $4(33.3)$ & 0 & $7(58.3)$ \\
\hline Obesidad & $3(20.0)$ & $3(25.0)$ & $4(66.7)$ & $2(16.7)$ \\
\hline \multicolumn{5}{|l|}{ Seguimiento postrasplante, $n(\%)$} \\
\hline $\begin{array}{l}\text { Meses desde trasplante, } \\
\text { media [IQR] }\end{array}$ & $65[36-276]$ & $\begin{array}{c}56.5 \\
{[24.7-83.5]}\end{array}$ & $\begin{array}{c}76 \\
{[55.5-154.5]}\end{array}$ & $\begin{array}{c}138 \\
{[38-198]}\end{array}$ \\
\hline Historia de rechazo & 0 & $1(8.3)$ & $1(16.7)$ & $2(16.7)$ \\
\hline $\begin{array}{l}\text { Creatinina basal, mg/ } \\
\mathrm{dL} \text {, mediana [IQR] }\end{array}$ & $\begin{array}{l}1.2 \\
{[1.0-1.7]}\end{array}$ & $\begin{array}{l}1.4 \\
{[0.97-1.7]}\end{array}$ & $\begin{array}{l}1.6 \\
{[1.3-2.5]}\end{array}$ & $\begin{array}{l}1.8 \\
{[1.3-2.5]}\end{array}$ \\
\hline \multicolumn{5}{|c|}{ Presentación clínica y evolución, $n(\%)$} \\
\hline \multicolumn{5}{|l|}{ Lugar de atención } \\
\hline Ambulatorio & $8(53.3)$ & $3(25.0)$ & 0 & 0 \\
\hline Sala general & $7(46.7)$ & $8(66.7)$ & $6(100.0)$ & $5(41.7)$ \\
\hline $\mathrm{UCl}$ & 0 & $1(8.3)$ & 0 & $7(58.3)$ \\
\hline \multicolumn{5}{|l|}{ Severidad por tomografía } \\
\hline No disponible & $1(6.7)$ & $4(33.3)$ & $2(33.3)$ & $1(8.3)$ \\
\hline Normal/leve $(<20 \%)$ & $7(46.7)$ & $3(25.0)$ & 0 & $1(8.3)$ \\
\hline Moderada (20-50\%) & $6(40.0)$ & $2(16.7)$ & $4(66.7)$ & $2(16.7)$ \\
\hline Severa $(>50 \%)$ & $1(6.7)$ & $3(25.0)$ & 0 & $8(66.7)$ \\
\hline $\begin{array}{l}\text { Días de hospitalización, } \\
\text { mediana [IQR] }\end{array}$ & $\begin{array}{c}6.5 \\
{[4.75-10]}\end{array}$ & $8[4-11]$ & $9.5[7.7-13]$ & $11[6-17]$ \\
\hline \multicolumn{5}{|l|}{ Ajuste de la inmunosupresión } \\
\hline $\begin{array}{l}\text { Inmunosupresión } \\
\text { usual mantenida }\end{array}$ & $6(40)$ & $5(41.7)$ & 0 & $2(16.7)$ \\
\hline $\begin{array}{l}\text { Suspensión total de } \\
\text { inmunosupresión* }\end{array}$ & $5(33.3)$ & $3(25.0)$ & $2(33.3)$ & $7(58.3)$ \\
\hline Reducción de inmunosupresión & 0 & 0 & 0 & 0 \\
\hline Retiro de antimetabolito & $3(20.0)$ & $1(8.3)$ & $1(16.7)$ & $1(8.3)$ \\
\hline Reducción de antimetabolito & 0 & $3(25)$ & $3(50)$ & $1(8.3)$ \\
\hline $\begin{array}{l}\text { Reducción de ICN } \\
\text { y antimetabolito }\end{array}$ & $1(6.7)$ & 0 & 0 & 0 \\
\hline Disminución de ICN & 0 & 0 & 0 & $1(8.3)$ \\
\hline \multicolumn{5}{|l|}{ Desenlaces, $\mathbf{n}(\%)$} \\
\hline Recuperación parcial & - & $1(8.3)$ & $2(33.3)$ & $2(16.7)$ \\
\hline Recuperación total & - & $10(83.3)$ & $4(66.7)$ & $6(50.0)$ \\
\hline Intubación & 0 & 0 & 0 & $6(50.0)$ \\
\hline Mortalidad & $1(6.7)$ & $1(8.3)$ & 0 & $4(33.3)$ \\
\hline
\end{tabular}

\section{Jueves 29}

12:10-12:40

\section{5}

Impacto de la presencia de alteraciones limítrofes en biopsias de vigilancia sobre los desenlaces del injerto renal

Fernández-Camargo Dheni A, Marino Lluvia, Muñiz-Cuervo Estela, Aceves-Rodríguez E Mauricio, Vargas Nancy J, Uribe Norma, Morales-Buenrostro Luis E

Nefrología \& Metabolismo Mineral y Anatomía Patológica. Instituto Nacional de Ciencias Médicas y Nutrición «Salvador Zubirán», CDMX, México.

Introducción: Las alteraciones limítrofes $(\mathrm{AL})$ se definen como cambios insuficientes para el diagnóstico de rechazo agudo leve y se pre- 
sentan en el 12 al 34\% de las biopsias de vigilancia realizadas durante el primer año postrasplante. Sin embargo, aún no se ha logrado determinar el efecto que tiene la presencia de AL en los desenlaces del injerto, especialmente en aquéllas que no se acompañan con disfunción renal. Material y métodos: En este estudio retrospectivo se incluyeron 40 biopsias de vigilancia de tres y 12 meses, de pacientes trasplantados renales con diagnóstico de AL y como controles incluímos 20 con rechazo celular agudo $(R C A)$ y 20 normales $(N)$, realizadas de enero de 2004 a marzo del 2020. Las biopsias con AL se dividieron en dos grupos: $A L$ disfusas (AL-D) $(n=20)$ cuando presentaban $>5$ túbulos con tubulitis, de lo contrario se clasificaron como AL focales (AL-F) ( $n=$ 20). Se excluyeron todas aquellas biopsias con FIAT > 50\% y cualquier diagnóstico al momento de la biopsia o previo que tuviera impacto en el pronóstico. Debido a la baja frecuencia de biopsias con RCA subclínico, se incluyeron ocho con RCA con disfunción del injerto al momento de la biopsia. Como puntos pronósticos se evaluaron las diferencias en la función renal, desarrollo de rechazo, pérdida del injerto, muerte del paciente y desarrollo de ADE de novo durante el primer año posterior a la biopsia. Resultados: Las características demográficas y del trasplante fueron similares entre los cuatro grupos. La mediana del tiempo del trasplante a la biopsia fue de 188 (98-157) días, sin ser diferente entre grupos $(p=0.412)$. El $90 \%$ de los pacientes en el grupo RCA recibieron tratamiento al momento de la biopsia, comparado con el 50 y $30 \%$ en los grupos AL-D y AL-F, respectivamente. Para la evaluación de los desenlaces, se realizó un subanálisis, dividiendo el grupo de RCA de acuerdo con la presencia de disfunción renal al momento de la biopsia. Se calculó el $\triangle T F G$ (TFG un año - TFG basal) sin encontrar diferencias significativas entre grupos; sin embargo, el grupo con RCA con disfunción (RCA-D) presentó más biopsias subsecuentes con rechazo $(p=0.0015)$ (Figura 1). Únicamente un paciente del grupo AL-D y tres del grupo RCA perdieron el injerto. Ningún paciente falleció durante el primer año de seguimiento. Discusión y conclusiones: Las AL son un diagnóstico frecuente en las biopsias de vigilancia de injertos renales. Únicamente el $40 \%$ de éstas recibieron tratamiento, en comparación al $90 \%$ de los pacientes con RCA. No se observaron diferencias en el cambio de la TFG durante el primer año de seguimiento; sin embargo, presentan una menor incidencia de rechazos en biopsias subsecuentes, en comparación con el grupo con RCA.

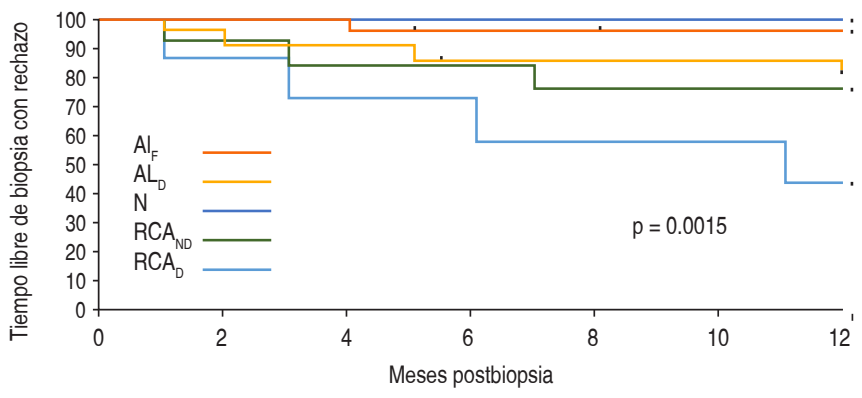

Figura 1: Presencia de rechazo en una biopsia subsecuente durante el primer año posterior a la biopsia.

Introducción: En la actualidad, enfrentamos una pandemia causada por el coronavirus 2, el síndrome respiratorio agudo (SARS-CoV-2). Los receptores de trasplante renal son considerados un grupo vulnerable por las comorbilidades asociadas e inmunosupresión crónica. Material y métodos: Estudio observacional donde se incluyeron 12 receptores renales con diagnóstico de neumonía por SARS-CoV-2 en el Hospital de Especialidades «Dr. Antonio Fraga Mouret» Centro Médico Nacional «La Raza». Debido al pequeño tamaño de la muestra, únicamente se empleó estadística descriptiva para las características clínicas principales y resultados a corto plazo. Resultados: De los 12 pacientes ingresados con neumonía por SARS-CoV-2, fueron siete $(58 \%)$ del sexo masculino y cinco $(42 \%)$ del femenino. El rango de edad fue de 26-69 años, y en tiempo después del trasplante renal fue de 3 meses-30 años. Los injertos renales fueron siete (58\%) de donante fallecido y cinco (42\%) de vivo. En cuanto a las comorbilidades, 11 (92\%) pacientes tenían hipertensión arterial, dos (17\%) diabetes mellitus y seis (50\%) obesidad. Los regímenes de inmunosupresión de mantenimiento incluyeron tacrolimus en siete (58\%) pacientes, dos (17\%) con ciclosporina, dos (17\%) con sirolimus, ocho (67\%) con micofenolato de mofetilo, cuatro (33\%) con azatioprina y los $12(100 \%)$ con prednisona; en los ajustes en la inmunosupresión durante su hospitalización, en seis pacientes se suspendieron y en cuatro pacientes se redujo la dosis, en 10 se administró esteroide intravenoso, seis $(60 \%)$ dexametasona y cuatro $(40 \%)$ hidrocortisona. En relación a los síntomas más frecuentes, todos cursaron con fiebre, $11(92 \%)$ con disnea, $10(83 \%)$ con dolor torácico, nueve $(75 \%)$ con mialgias y artralgias, siete (58\%) con tos, cinco $(42 \%)$ con cefalea, cinco $(42 \%)$ con odinofagia, tres (25\%) con diarrea, el tiempo de estancia intrahospitalaria promedio fue de siete días (1-14 días), nueve (75\%) padecieron linfopenia. Todos los pacientes tuvieron hallazgos de lesiones sugestivas de neumonía por SARS-CoV-2 moderada y grave por tomografía computarizada. En nueve $(75 \%)$ presentaron disfunción aguda del injerto, de los cuales dos (22\%) presentaban glomerulopatía crónica del trasplante y requirieron hemodiálisis. Todos los pacientes recibieron oxígeno complementario, y seis $(50 \%)$ requirieron ventilación mecánica asistida. En cuanto a la evolución, seis $(50 \%)$ fueron egresados a domicilio por evolución satisfactoria y seis $(50 \%)$ fallecieron. Discusión y conclusiones: Presentamos las características clínicas y resultados de 12 receptores de trasplante renal con infección por SARS-CoV-2 confirmada por laboratorio (RTqPCR SARS-CoV-2). Todos fueron ingresados en nuestra unidad por cursar con una neumonía progresiva, encontrando una mortalidad general del 50 y $100 \%$ en los pacientes que requirieron ventilación mecánica asistida. Aunque nuestros hallazgos son preliminares y cuenta con las limitaciones en el tamaño de la muestra y el tiempo de seguimiento es corto, creemos que proporcionan una referencia de utilidad en este tipo de pacientes.

07

12:30-12:40
Impacto de conversión hospitalaria para atención de COVID-19 en el Programa de Trasplante Renal del INCMNSZ

Valdés Lagunes David Alfonso, Morales Buenrostro Luis Eduardo Instituto Nacional de Ciencias Médicas y Nutrición «Salvador Zubirán».
06 Receptores de trasplante renal ingresados por neumonía por SARS

12:20-12:30 CoV-2. Reporte de 12 casos confirmados en UMAE HE CMN «La Raza»

Robledo-Meléndez Arlette, Cruz-Santiago José, Noriega-Salas Lorena, García-Jiménez Catalina de Rosario, Meza-Jiménez Guillermo, Bernáldez-Gómez Germán

Departamento de Trasplantes HE CMN «La Raza», IMSS, CDMX, México.
Introducción: En México, se decidió que el Instituto Nacional de Ciencias Médicas y Nutrición «Salvador Zubirán» (INCMNSZ) sufriera conversión para atender exclusivamente pacientes con COVID-19. Con esto, muchas actividades asistenciales y de investigación tuvieron que parar. Paró por completo la actividad de trasplante renal de donador vivo y fallecido, la realización de biopsias de injerto renal y quedó cancelada la consulta externa, ofreciendo atención telefónica a pacientes trasplantados, dando seguimiento presencial sólo a pacientes trasplantados en las semanas previas a la contingencia. Objetivo: Re- 
portar el tipo de actividades del Programa de Trasplante Renal que se vieron afectadas por la conversión hospitalaria y su magnitud. Material y métodos: Estudiaremos la actividad del Programa de Trasplante Renal del INCMNSZ durante el periodo de conversión. Se revisará la base de datos del departamento, en la cual se registran los trasplantes de riñón tanto de donador vivo como fallecido. Se compararán con los trasplantes realizados en el año previo a la conversión hospitalaria, durante el mismo periodo a evaluar. Se lleva un registro de biopsias, determinaremos el número de biopsias esperadas en el periodo y el número de biopsias realizadas, para obtener la reducción. Respecto a la actividad de las diferentes consultas del programa de trasplantes, se tiene el registro de consultas realizadas el año previo y durante el periodo de conversión hospitalaria, así podremos obtener el impacto en este aspecto. Se revisarán las limitaciones al programa de trasplante hasta el cierre del estudio o hasta que se reanude la actividad de trasplantes en el INCMNSZ. Resultados: La actividad de trasplante renal tanto de donador vivo como fallecido fue suspendida totalmente a partir del 18 de marzo de 2020 a la fecha, lo que representa 23 eventos de trasplante vivo que se hubieran llevado a cabo en ese periodo. Se cancelaron 50 biopsias de injerto que estaban programadas en un periodo de dos meses. En relación a la consulta externa, únicamente se atendieron 40 pacientes de 651 que estaban citados originalmente, en un periodo de 16 semanas (Tabla 1). Hasta el momento tenemos identificados a dos pacientes que han tenido eventos de rechazo agudo grave, uno de ellos resultó en pérdida del injerto, y se están estudiando 14 pacientes más con deterioro agudo de la función del injerto. Conclusiones: La conversión hospitalaria por COVID-19 ha detenido por completo la actividad de trasplante renal del INCMNSZ. Las biopsias de injerto estuvieron detenidas por completo durante cuatro meses, reanudándose al $25 \%$ de lo habitual. Más del $90 \%$ de los pacientes programados a consulta externa perdieron su cita.

Tabla 1: Actividades del programa de trasplantes que se vieron afectadas.

\begin{tabular}{|lccc}
\hline \multicolumn{1}{c}{ Tipo de actividad } & $\begin{array}{c}\text { Realizadas } \\
\text { marzo-julio 2019 }\end{array}$ & $\begin{array}{c}\text { Realizadas } \\
\text { marzo-julio 2020 }\end{array}$ & \% reducción \\
\hline TRDV & 14 & 0 & 100 \\
TRDC & 9 & 0 & 100 \\
Biopsias injerto & 122 & 0 & 100 \\
Consulta seguimiento & 896 & 40 & 95 \\
Preconsulta & 132 & 0 & 100 \\
Plasmaféresis & 16 & 0 & 100 \\
Administración ambulatoria & 28 & 6 & 80 \\
de rituximab, IglV & & & \\
\hline
\end{tabular}

\section{8}

12:40-12:50

\section{Enfermedad por COVID-19 en receptores} de trasplante de órgano sólido: reporte multicéntrico de la experiencia mexicana

Sánchez-Cárdenas Mónica, ${ }^{*}$ Vásquez-Jiménez Enzo, ${ }^{\ddagger}$

Velázquez-Silva Ricardo Iván, ${ }^{*}$ Vilatobá-Chapa Mario, $\$$

Gómez-Navarro Benjamín," Sánchez-Macías Lucio Octavio,"

Rodríguez-Chagolla José Manuel, ${ }^{* *}$ García-Juárez Ignacio, ${ }^{\ddagger}$

Abraham-Mancilla Severo Manuel,,$\S$ Flores-Gama César, $\neq$

Parra-Ávila Idalia, "17 Morales-Buenrostro Luis Eduardo*

* Nefrología del Trasplante. Departamento de Nefrología y Metabolismo Mineral. INCMNSZ. ₹ Departamento de Nefrología, INCICh. ^ Departamento de Trasplante, INCMNSZ. " Departamento de Nefrología. CMNO, IMSS. Guadalajara. " Hospital San Javier, Guadalajara, México. ${ }^{* \star}$ Departamento de Nefrología y Medicina Interna. Centro Médico ISSEMyM, Toluca. \#Departamento de Gastroenterología. INCMNSZ. ${ }^{\$ \S}$ Departamento de Trasplante. UMAE, Centro Médico Nacional del Bajío. " ${ }^{\text {Tn }}$ Departamento de Trasplante. UMAE, IMSS. Mérida, Yucatán, México.

Introducción: Los receptores de trasplante de órgano sólido, dado el estado de inmunosupresión basal y la amplia comorbilidad, parecen encontrarse en riesgo particularmente alto de infección por COVID-19
Tabla 1 (08): Características basales y evolución clínica de los receptores de trasplante de órgano sólido ( $\mathrm{N}=59$ ).

\begin{tabular}{|c|c|c|c|c|c|}
\hline & \multicolumn{4}{|c|}{ Receptores riñón } & \multirow{2}{*}{$\begin{array}{c}\begin{array}{c}\text { Receptores } \\
\text { higado }\end{array} \\
\begin{array}{c}\text { Total higado } \\
n=10\end{array}\end{array}$} \\
\hline & $\begin{array}{l}\text { Ambulatorios } \\
n=12\end{array}$ & $\begin{array}{l}\text { Hospitalizados } \\
n=29\end{array}$ & $\begin{array}{l}\text { UCl } \\
n=8\end{array}$ & $\begin{array}{l}\text { Total } \\
\text { riñón } \\
n=49\end{array}$ & \\
\hline \multicolumn{6}{|c|}{ Características clínicas y demográficas, $n$ (\%) } \\
\hline Edad, mediana (IQR) & $43(29.2-49)$ & $45(34-53.5)$ & $43(34-53.7)$ & $43(34-52.5)$ & $50(43-53)$ \\
\hline Género masculino & $6(50.0)$ & $15(51.7)$ & $6(75)$ & $27(55.1)$ & $6(60)$ \\
\hline \multicolumn{6}{|l|}{ Comorbilidad, $n(\%)$} \\
\hline Diabetes & $2(16.7)$ & $10(34.5)$ & $1(12.5)$ & $13(26.5)$ & $6(60)$ \\
\hline Hipertensión & $6(50.0)$ & $14(48.3)$ & $7(87.5)$ & $27(55.1)$ & $1(10)$ \\
\hline Sobrepeso & $6(50.0)$ & $8(27.6)$ & $5(62.5)$ & $19(38.8)$ & $4(40)$ \\
\hline Obesidad & $1(8.3)$ & $11(37.9)$ & $1(12.5)$ & $13(26.5)$ & $2(20)$ \\
\hline \multicolumn{6}{|c|}{ Seguimiento postrasplante } \\
\hline $\begin{array}{l}\text { Meses desde } \\
\text { trasplante, } \\
\text { media (IQR) }\end{array}$ & $56.5(12.7-261)$ & $82(56-204)$ & $51.5(23.2-174)$ & & $54(24-72)$ \\
\hline $\begin{array}{l}\text { Historia de } \\
\text { rechazo, } \mathrm{n}(\%)\end{array}$ & 0 & $3(8.8)$ & $1(12.5)$ & $4(8.3)$ & 0 \\
\hline $\begin{array}{l}\text { Creatinina basal, mg/ } \\
\mathrm{dL} \text {, mediana (IQR) }\end{array}$ & $1.2(0.9-1.73)$ & $1.6(1.29-2.0)$ & $1.37(1.0-2.4)$ & $1.4(0.96-1.8)$ & $0.9(0.8-1.2)$ \\
\hline \multicolumn{6}{|c|}{ Presentación clínica y evolución } \\
\hline $\begin{array}{l}\text { Días de evolución, } \\
\text { mediana (IQR) }\end{array}$ & $7(3-7)$ & $7(3-9)$ & $7(5.5-10.2)$ & $7(3.5-9)$ & $5(4-8)$ \\
\hline \multicolumn{6}{|l|}{$\begin{array}{l}\text { Síntoma de } \\
\text { presentación, n (\%) }\end{array}$} \\
\hline Fiebre & $13(76.5)$ & $30(8.2)$ & $6(75.0)$ & $41(83.7)$ & $8(80)$ \\
\hline Diarrea & $3(17.6)$ & $14(41.2)$ & 0 & $16(26.5)$ & $1(10)$ \\
\hline Tos & $6(35.3)$ & $23(67.6)$ & $7(87.5)$ & $28(57.1)$ & $8(80)$ \\
\hline Cefalea & $10(58.8)$ & $22(64.7)$ & $5(62.5)$ & $30(61.2)$ & $7(70)$ \\
\hline \multicolumn{6}{|l|}{$\begin{array}{l}\text { Severidad por } \\
\text { tomografía, } n(\%)\end{array}$} \\
\hline No disponible & $4(33.3)$ & $4(13.7)$ & $1(12.5)$ & $9(16.3)$ & 0 \\
\hline Normal/leve (<20\%) & $7(58.3)$ & $3(10.3)$ & $1(12.5)$ & $11(2.4)$ & $8(80)$ \\
\hline Moderada (20-50\%) & $1(8.3)$ & $15(5.1)$ & 0 & $16(32.6)$ & 0 \\
\hline Severa $(>50 \%)$ & 0 & $7(24.1)$ & $6(75)$ & $13(26.5)$ & $2(20)$ \\
\hline \multicolumn{6}{|l|}{$\begin{array}{l}\text { Lesión renal } \\
\text { aguda, } n(\%)^{\star}\end{array}$} \\
\hline Estadio 1 & $3(25)$ & $10(34.5)$ & $1(12.5)$ & $14(28.6)$ & $1(10)$ \\
\hline Estadio 2 & 0 & $7(24.1)$ & 0 & $7(14.3)$ & 0 \\
\hline Estadio 3 & 0 & $5(17.2)$ & $7(87.5)$ & $12(24.5)$ & 0 \\
\hline $\begin{array}{l}\text { Días de } \\
\text { hospitalización, } \\
\text { mediana (IQR) }\end{array}$ & 0 & $8(5-10)$ & $12.5(7.5-29.7)$ & $8(6-12)$ & $6(4-10)$ \\
\hline \multicolumn{6}{|c|}{ Ajuste de la inmunosupresión } \\
\hline $\begin{array}{l}\text { Inmunosupresión } \\
\text { usual mantenida }\end{array}$ & $10(83.3)$ & $4(13.8)$ & 0 & $14(28.5)$ & $7(70)$ \\
\hline $\begin{array}{l}\text { Suspensión total de } \\
\text { inmunosupresión }{ }^{\ddagger}\end{array}$ & 0 & $12(41.4)$ & $5(62.5)$ & $17(34.7)$ & 0 \\
\hline \multicolumn{6}{|l|}{$\begin{array}{l}\text { Reducción de } \\
\text { inmunosupresión }\end{array}$} \\
\hline $\begin{array}{l}\text { Retiro de } \\
\text { antimetabolito }\end{array}$ & $1(8.3)$ & $5(17.2)$ & $1(12.5)$ & $7(14.3)$ & $2(20)$ \\
\hline $\begin{array}{l}\text { Reducción de } \\
\text { antimetabolito }\end{array}$ & 0 & $6(20.7)$ & $1(12.5)$ & $7(14.3)$ & 0 \\
\hline $\begin{array}{l}\text { Reducción de ICN } \\
\text { y antimetabolito }\end{array}$ & $1(8.3)$ & $2(6.9)$ & 0 & $3(6.1)$ & $1(10)$ \\
\hline Reducción de ICN & 0 & 0 & $1(12.5)$ & $1(2.0)$ & \\
\hline \multicolumn{6}{|l|}{ Desenlaces, $n(\%)$} \\
\hline Lesión renal aguda & $3(25)$ & $22(75.8)$ & $8(100.0)$ & $33(67.0)$ & $1(10)$ \\
\hline $\begin{array}{l}\text { Recuperación } \\
\text { parcial }\end{array}$ & $1(33)$ & $4(18.1))$ & 0 & $5(15.1)$ & 0 \\
\hline Recuperación total & $2(66)$ & $16(72.7)$ & $5(62.5)$ & $23(69.6)$ & 1 \\
\hline $\begin{array}{l}\text { Terapia de } \\
\text { reemplazo renal }\end{array}$ & 0 & $1(4.5)$ & $5(62.5)$ & $56(12.2)$ & 0 \\
\hline Intubación & 0 & 0 & 0 & $6(12.2)$ & 0 \\
\hline Mortalidad & 0 & $3(10.3)$ & $3(37.5)$ & $6(12.2)$ & 0 \\
\hline
\end{tabular}

* Definido por los criterios de Kidney Disease: Improving Global Outcomes (KDIGO), ₹ Sólo uso de prednisona. $\mathrm{UCI}=$ Unidad de Cuidados Intensivos, IQR = rango intercuartil, ICN = Inhibidor de calcineurina.

y sus complicaciones. Reportamos la evolución clínica y desenlaces a corto plazo de receptores de trasplante renal y hepático tratados en seis centros de Atención de Tercer Nivel en México. Material y métodos: Evaluamos de manera retrospectiva aquellos receptores de trasplante renal y hepático mayores de 18 años, con diagnóstico con- 
firmado de infección por COVID-19 tratados en seis centros de tercer nivel en México. Resultados: Datos de 49 receptores de trasplante renal y 10 receptores de trasplante hepático fueron registrados. La mediana de edad global fue de 45 años (IQR 36-53). Las comorbilidades más frecuentes fueron sobrepeso-obesidad presentes en 39 pacientes $(66.1 \%)$, hipertensión $28(47.5 \%)$ y diabetes $19(32.2 \%)$. En los receptores de trasplante renal fue requerido proceder a hospitalización en sala general en 29 casos (59.1\%), con ingreso a la Unidad de Terapia Intensiva en ocho pacientes (16.3\%). La media de días de hospitalización fue de ocho días (IQR 6-12). Doce pacientes $(24.4 \%)$ fueron manejados de manera ambulatoria. Se documentó lesión renal aguda en 33 casos (66.7\%), de los cuales seis (18.1\%) requirieron manejo dialítico. La mortalidad global fue de $12.2 \%$ y la mortalidad en $\mathrm{UCl}$ de $37.5 \%$. Las características demográficas basales y el manejo clínico llevado a cabo pueden observarse en la Tabla 1. Adicionalmente, datos de 10 receptores de trasplante hepático fueron incluidos. Durante el seguimiento $5 / 10$ requirieron hospitalización y no hubo fallecimientos en este grupo de pacientes. La inmunosupresión habitual fue mantenida en 7/10 casos. Discusión y conclusión: Similar a lo reportado en la literatura, los receptores de trasplante de órgano sólido tienen una evolución clínica muy heterogénea; algunos pacientes cursan con evolución benigna a pesar del estado de inmunosupresión crónica y múltiples comorbilidades. No existen recomendaciones universales en cuanto a los ajustes de inmunosupresión a realizar durante el evento agudo, pero debe buscarse un balance entre contener el riesgo de rechazo por retiro de medicación y evitar complicaciones infecciosas por sobreinfección.

\section{Viernes 30}

\section{2:10-12:30}

\section{9}

12:10-12:20

Experiencia de los primeros 5 casos de trasplante dual en el Instituto Nacional de Nutrición «Salvador Zubirán»

Torres del Real Daniel Ángel, Parmentier de León Catherine, Romero Morelos Ricardo Daniel, Carpinteyro Espín Paulina, Chávez Villa Mariana, Navarro del Río Estephania, Cruz Martínez Rodrigo, Contreras Saldivar Alan, Vilatobá Chapa Mario Instituto Nacional de Ciencias Médicas y Nutrición «Salvador Zubirán».

El primer / trasplante dual de riñón (DKT) se realizó en 1996 en los Estados Unidos. Bajo la «Teoría de masa»: existencia de una doble masa de nefronas compensaría el desequilibrio entre la masa de nefronas limitada de riñones de donante de criterios extendidos (ECD) y las necesidades fisiológicas del trasplantado. Los aspectos quirúrgicos a considerar son: condiciones vasculares previas donante y receptor, alto riesgo de calcificaciones vasculares, resistencias vasculares intraparenquimatosas aumentadas, riesgo de trombosis del injerto. Objetivo: Exponer la experiencia del Instituto Nacional de Ciencias Médicas y Nutrición «Salvador Zubirán» (INCMNSZ) en trasplante renal dual. Resultados: En total se han realizado cinco trasplantes renales duales del 2017 a la fecha, se diseñó un algoritmo para la toma de decisiones en riñones con Remuzzi 5 y 6 y se ajustó la edad del donador para recibir este tipo de trasplante. De los 5 casos, el tiempo quirúrgico medio son 401 min (210-495), el tiempo de isquemia fría 1,043 min. (897-1452), isquemia tibia 46.6 minutos (32-66 min), y la media de tiempo para el inicio de la uresis fue de 21.6 minutos (16-44). Dos pacientes (40\%) presentaron retardo en la función del injerto y en cuanto a las complicaciones se presentaron dos complicaciones Clavien IIIb y una Clavien V y Clavien IIIb. En cuanto a los receptores, $60 \%$ corresponden al sexo masculino y la edad promedio fue de 62 años (43-72 años), en cuatro pacientes el
Remuzzi del injerto fue de 5. Conclusiones: El trasplante dual es una opción factible en el contexto del paciente añoso con enfermedad renal, como tal se trata de pacientes cuya posibilidad de recibir un órgano con criterios estándar son pocas y aunque las complicaciones a simple vista parecen presentarse en un porcentaje importante, a la larga los receptores presentan buena sobrevida.

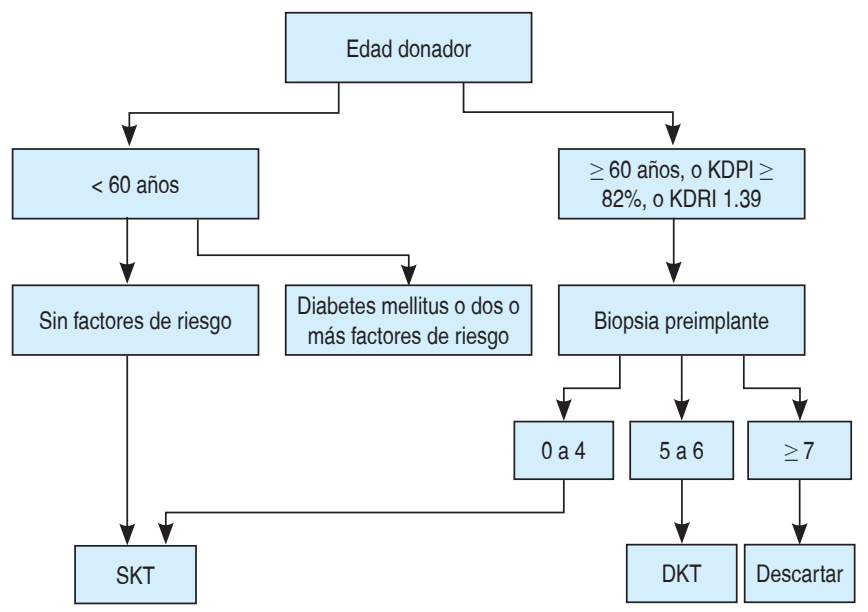

Si donador diabético se deberá realizar biopsia preimplante sin importar edad, KDRI o KDPI

Dos o más factores de riesgo:

- eGFr $\leq 60 \mathrm{~mL} / \mathrm{min}$ (CKD-EPI)

- Proteinuria $\geq 1 \mathrm{~g} / \mathrm{día}$

- Antecedentes de eventos cardiovasculares o muerte encefálica por evento cardiovascular

- Hipertensión arterial sistémica

- Uso de norepinefrina > 0.5 gammas

Figura 1: Algoritmo diseñado posterior al primer trasplante dual en Instituto Nacional de Ciencias Médicas y Nutrición «Salvador Zubirán».

$(\mathrm{DKT})=$ trasplante dual de riñón.
010

12:20-12:30
Progenitores hematopoyéticos provenientes de médula ósea pueden ayudar en la inducción de tolerancia en el trasplante de intestino delgado
Arreola Nidia Monserrat, ${ }^{*, \neq}$ Pascual-Miguel Bárbara, ${ }^{\S}$ Stringa Pablo," González-Navarro Pablo," Papa-Gobbi Rodrigo, * Vela María, ${ }^{\S}$ Muñoz-Fernández de Legaria Marta, ${ }^{* \star}$ Sanz Sandra, ${ }^{\ddagger}$ Camps Onys, ${ }^{\prime}$ Pérez-Martínez Antonio, ${ }^{\S}$ Hernández-Oliveros Francisco* * Hospital Universitario La Paz Instituto de Investigaciones en Salud (IDiPAZ), Grupo de Trasplantes-Cirugía Experimental. ₹ Hospital de Pediatría, Centro Médico Nacional de Occidente, Instituto Mexicano del Seguro Social, Guadalajara, México. \& Hospital Universitario La Paz Instituto de Investigaciones en Salud (IDiPAZ) Investigación traslacional en Oncología Pediátrica-Trasplante Hematopoyético y Terapia Celular. " Instituto de Estudios Inmunológicos y Fisiopatológicos (IIFP/CONICET)-UNLP. " FIB-Hospital Infantil Universitario Niño Jesús-Unidad de Terapia Celular y Genética. ${ }^{* \star}$ Laboratorio de Patología

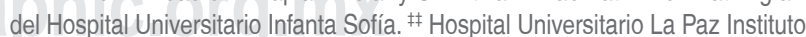
de Investigaciones en Salud (IDiPAZ), Laboratorio de Histopatología.

Introducción: Después de un trasplante, los pacientes deben recibir inmunosupresión de por vida (IS) para evitar que el sistema inmunológico ataque al órgano trasplantado. La inducción de tolerancia por infusión de progenitores hematopoyéticos se incluye entre las terapias avanzadas con gran potencial en el campo del trasplante de órganos sólidos. El objetivo de este estudio es inducir tolerancia por infusión diferida de progenitores hematopoyéticos 
en un modelo experimental de trasplante intestinal (IT). Material y métodos: Se utilizó un modelo animal de dos cepas alogénicas de ratas (BN para Lew), a las que se les realizó un IT con ileostomía terminal seguida de inyección intravenosa de $4 \times 10^{6}$ células de CD90.1+ progenitores criopreservados provenientes de fémur de cepa donante. Los progenitores de CD90.1+ se purificaron previamente mediante clasificación de células magnéticas (pureza $\geq$ $99 \%)$. Se utilizó IS mediante un esquema corto de FK506 durante $0+6$ días vía subcutánea después del IT. A través de la estoma abdominal se monitorizó el injerto los días 3, 7, 14, 21 y 28 post-IT (POD) así como extracción de sangre periférica. A nivel celular, el quimerismo de las células del donante (RT1Ac +, MHC-I del donante) en el receptor y los linfocitos $T$ reguladores (Treg, CD3 + CD4 + CD25 + FoxP3 +) como reguladores clave de la tolerancia del injerto se estudiaron mediante citometría de flujo; a nivel tisular, el estado del injerto se describió mediante histología (hematoxilina y eosina). Resultados y discusión: Se realizaron análisis y se compararon con grupo control (IT + FK506 sin inyección de progenitores $\mathrm{CD} 0^{+}$). Se encontró quimerismo hasta el día 14 POD y cayó casi a cero el día 21 POD. Mientras que el grupo de control tenía un máximo de células Treg el día siete y comenzó a disminuir el día 14 , el grupo inyectado con progenitores CD90.1 + «grupo CD90» mantuvo la tendencia al aumento durante una semana más, disminuyendo el día 21, coincidiendo exactamente con el aumento de linfocitos T CD8 + y la pérdida de quimerismo de células del donante en sangre. La puntuación de histopatología mostró diferencias significativas en el día 14 POD entre ambos grupos. Ambos grupos en 21 POD presentaron rechazo agudo. Conclusiones: La inyección diferida de progenitores $\mathrm{CD} 90^{+}$podría ayudar a la tolerancia del injerto durante más tiempo. Una segunda reinfusión de los progenitores CD90.1 + mejoraría el resultado de la terapia. Este estudio muestra un abordaje de una posible terapia celular para el manejo del rechazo del injerto en la TI.

\section{Comportamiento de la creatinina en pacientes trasplantados con infección por SARS-CoV-2} 12:30-12:40

Robledo Meléndez Arlette, ${ }^{*}$ Torres Arce Abril Monserrat, ${ }^{\ddagger}$ Espinoza Pérez Ramón, $\$$ Santos Caballero Marlene," Cruz Santiago José, ${ }^{*}$ Meza Jiménez Guillermo, ${ }^{*}$

Carreño Rodríguez Yasmín Rocío, ${ }^{*}$ Medina Uicab Carlos Jesús, ${ }^{*}$ Noriega Salas Lorena, ${ }^{*}$ Hernández Rivera Juan Carlos H" * UTR HE CMN «La Raza», CDMX, México. ₹ UTR UMAE Puebla, Puebla. § UTR HE CMN Siglo XXI, CDMX, México. ^ UTR HG CMN «La Raza", CDMX, México. "UIMEN HE CMN Siglo XXI, CDMX, México.
Introducción: Los pacientes trasplantados presentan como principales complicaciones para falla del injerto el rechazo y las infecciones; ante la actual pandemia de COVID-19 igualmente la disfunción aguda es una de las principales complicaciones observadas. El objetivo de este estudio es presentar el comportamiento de la creatinina en los pacientes trasplantados infectados con SARS-CoV-2. Material y métodos: Cohorte retrospectiva de 57 pacientes trasplantados con infección moderada y grave por SARS-CoV-2 de 3 unidades de trasplante renal. Se realiza análisis de creatinina basal, al ingreso, pico máximo de elevación y al final de la hospitalización, independientemente del desenlace por Friedman; con p significativa menor de 0.05 , programa estadístico SPSS versión 25. Resultados: De los 57 pacientes que acudieron atención médica, 29 de ellos (50.9\%) presentó disfunción aguda del injerto; siendo 25 desde su ingreso, los otros cuatro presentan elevación de azoados días posteriores al ingreso. 19 pacientes con respuesta a las medidas de hidratación y manejo de la infección y 10 con la necesidad de terapia de remplazo renal. Discusión y conclusiones: La disfunción aguda del injerto es una complicación común en el contexto del paciente trasplantado renal con infección asociada a coronavirus. Es fundamental la detección sobre todo desde el ingreso hospitalario que nos traduce labilidad de la función renal ante el SARS-CoV-2, y la detección oportuna es fundamental para las medidas de soporte hídrico, evitando sobretodo la necesidad de terapia de remplazo renal.

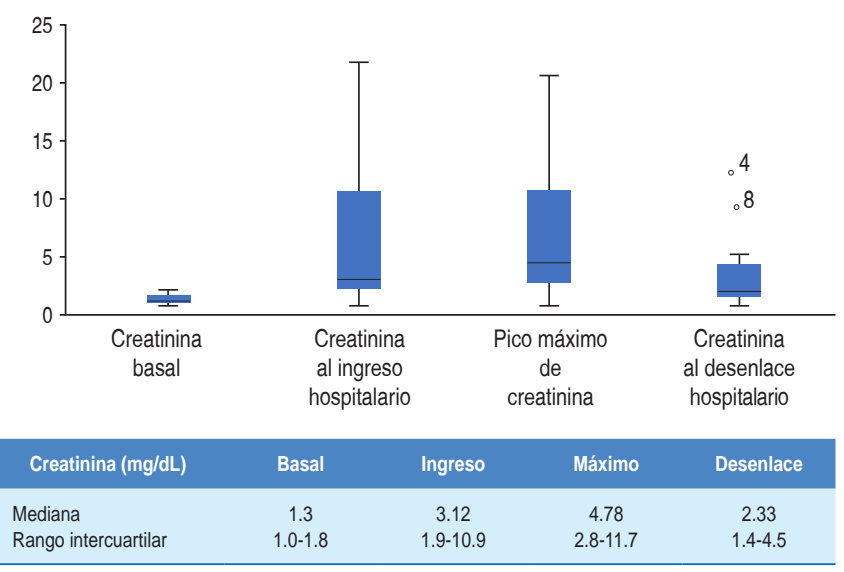

Figura 1: Comportamiento de la creatinina $(\mathrm{mg} / \mathrm{dL})$ en pacientes trasplantados que cursaron con COVID-19. 